\title{
Variation Trend of Vitamin D Status in Wintertime Among Infants: A Cross-sectional Study
}

\author{
Wei $\mathrm{Li}^{1,2, \dagger}$, Qiong Dai ${ }^{2,3, \dagger}$, Yongying Qiu ${ }^{4, \dagger}$, Jianqiong Liu ${ }^{2,5}$, Yan Guo ${ }^{6}$, Bin Wang ${ }^{7, *}$ \\ ${ }^{1}$ Department of Pharmacy, Maternal and Child Health Hospital of Hubei Province, Wuhan, China \\ ${ }^{2}$ Women and Children's Hospital of Hubei Province, Wuhan, China \\ ${ }^{3}$ Health Care Department, Maternal and Child Health Hospital of Hubei Province, Wuhan, China \\ ${ }^{4}$ Department of Gynaecology, Maternal and Child health Hospital of Xinfen County, Ganzhou, China \\ ${ }^{5}$ Department of Child Health Care, Maternal and Child Health Hospital of Hubei Province, Wuhan, China \\ ${ }^{6}$ Wuhan Centers for Disease Prevention and Control, Wuhan, China \\ ${ }^{7}$ Department of Laboratory of Tongji Hospital, Tongji Medical College, Huazhong University of Science and Technology, Wuhan, China \\ Email address: \\ liwei1988hust@126.com (Wei Li), daiqiong74@163.com (Qiong Dai),xfqyy@163.com (Yongying Qiu), \\ niuniu504231@163.com (Jianqiong Liu),250322883@qq.com (Yan Guo),912963506@qq.com (Bin Wang) \\ ${ }^{*}$ Corresponding author \\ $\dagger$ Wei Li, Qiong Dai and Yongying Qiu are co-first authors.
}

\section{To cite this article:}

Wei Li, Qiong Dai, Yongying Qiu, Jianqiong Liu, Yan Guo, Bin Wang. Variation Trend of Vitamin D Status in Wintertime Among Infants: A Cross-sectional Study. Journal of Gynecology and Obstetrics. Vol. 7, No. 5, 2019, pp. 149-154. doi: 10.11648/j.jgo.20190705.16

Received: September 8, 2019; Accepted: September 25, 2019; Published: October 10, 2019

\begin{abstract}
Background The requirement of vitamin D in different stage may be diverse, but research on the variation trend of vitamin D status during infancy is scarce. Objective This study aimed to explore the variation trend of vitamin D status and influencing factors of vitamin D deficiency and insufficiency during wintertime among infants. Methods A cross-sectional study was conducted. A total of 500 infants were included in our study during January to March 2017. The serum 25(OH)D was performed by chemiluminescent assay. Multivariate Logistic regression was conducted to evaluate the influencing factors of vitamin D deficiency and vitamin D insufficiency. Results The older infants had a higher concentration of vitamin D than younger infants $(\mathrm{r}=0.62, P<0.0001)$. And the overall prevalence of vitamin $\mathrm{D}$ deficiency and insufficiency were $33.80 \%$ and $18.00 \%$, respectively. With the increase of month age, the risk of vitamin D deficiency $(\mathrm{OR}=0.63,95 \% \mathrm{CI}: 0.57-0.70)$ and insufficiency ( $\mathrm{OR}=0.69,95 \% \mathrm{CI}$ : 0.62-0.77) were both declined. In addition, the infant with low birthweight, breastfed, living in rural area were correlated with a higher risk of vitamin D deficiency and insufficiency $(P<0.05)$. Conclusion Vitamin $\mathrm{D}$ deficiency and insufficiency prevalence changed with month age, routine monitoring of vitamin D levels is necessary for infant.
\end{abstract}

Keywords: Vitamin D Status, Vitamin D Deficiency, Vitamin D Supplementation, Infant, Wintertime, Breastfed

\section{Introduction}

Vitamin D, which can enhance intestinal calcium and phosphate absorption and promote bone mineralization, has been identified as one of the key nutrients that contributes to the development and maintenance of optimum bone mass from the very early stage. [1] Without controversy, vitamin D deficiency with or without calcium deficiency, can lead to nutritional rickets, calcium homeostasis disturbance and osteomalacia. [2, 3] In addition, over the last two decades, with the discovery of vitamin $\mathrm{D}$ receptors in other body tissues and cells, the associations between vitamin D and several health outcomes, such as birthweight, dental caries, myopia and so on, have also been confirmed [4]. Vitamin D nutritional status has attracted more and more researchers' attention 
nowadays.

Infancy period is the fastest growth stage that needs a great dose of calcium and phosphate for osteogenesis. The infant has a relative high demand for vitamin D. In humans, the source of vitamin D mainly depends on skin exposure to ultraviolet B radiation. But for infant, the body surface area and frequency of exposure to sunlight are not enough to produce adequate vitamin $\mathrm{D}$, especially in winter. In addition, the concentration of vitamin D in breast milk is low, hence the dietary sources vitamin $\mathrm{D}$ become more limited. Vitamin D deficiency or insufficiency is vulnerable in infant [5].

Multiple guidelines or public health policies on vitamin D supplementation have been published in the last decade [6]. In 2008, American Academy of Pediatrics recommended that all infants should have a minimum intake of 400IU/day vitamin D supplement beginning soon after birth [7]. And in China, this recommendation has been in place since 2010 [8]. But with the aggravation of air pollution, urbanization, over-crowding, the endogenous synthesis of vitamin D might be less and less because of the reduced ultraviolet $\mathrm{B}$ radiation exposure time and frequency in recent years [9]. Seasonal variation of sunlight is one of critical factors in the determination of human vitamin D, and endogenous synthesis of vitamin D is even worse in winter. The implementation effect needs to be evaluated in the current environment, especially in winter. In addition, the requirement of vitamin $\mathrm{D}$ in different stage may be diverse, but research on the variation trend of vitamin $\mathrm{D}$ status during infancy is scarce [10]. We conducted a cross-sectional study to measure the wintertime vitamin D status of infant. The aim of our study was conducted to (1) explore the variation trend of vitamin D status with month of age (2) explore the influencing factors of the vitamin D deficiency and insufficiency.

\section{Materials and Methods}

\subsection{Study Participants}

Our study was designed as a cross-sectional study. A total of 500 infants were consecutively recruited in department of pediatrics, Tongji Hospital of Huazhong University of Science \& Technology, Wuhan city, central China, during January to March 2017. Exclusion criteria were: age $>12$ months; birth defects; chronic disease; use of medications known to affect vitamin $\mathrm{D}$ metabolism. The study was approved by the institutional review boards of Tongji Medical College, Huazhong University of Science \& Technology, and parents or guardians provided written informed consent at study enrollment.

\subsection{Demographic Indices}

The demographic data (including sex, month age, birthweight, region of residence, feeding patterns and so on) were collected from the parents or guardians (most preferably from the mother) during the first visit to the hospital. All interviews were conducted by the trained nurses with the use of a standardized questionnaire.

\subsection{Laboratory Measurements}

$2 \mathrm{~mL}$ blood sample was obtained for each participant accompany with the routine blood drawing. Serum 25(OH)D test was performed in the hospital laboratory using chemiluminescent assay. The infants were divided into 3 groups using the cut-off values proposed by the Endocrine Society: vitamin D deficiency (serum 25(OH)D concentrations $\leq 20 \mathrm{ng} / \mathrm{mL}$ ), vitamin $\mathrm{D}$ insufficiency (serum $25(\mathrm{OH}) \mathrm{D}$ concentrations $>20$ but $<30 \mathrm{ng} / \mathrm{mL}$ ) and optimal vitamin D status (serum $25(\mathrm{OH}) \mathrm{D}$ concentrations $\geq 30$ $\mathrm{ng} / \mathrm{mL})$.

\subsection{Statistical Analysis}

Normality of distribution for continuous variables was tested by the Kolmogorov-Smirnov test. Normal distribution data were presented as mean $\pm \mathrm{SD}$, and the differences among groups were compared by analysis of variance (ANOVA). For categorical data, the difference between groups were tested using the chi-square test. Spearman rank correlation was used to calculated correlations between serum $25(\mathrm{OH}) \mathrm{D}$ level and month age. The multivariate Logistic regression with stepwise selection was preformed to evaluate the influencing factors of vitamin $D$ deficiency and vitamin D insufficiency. $P<0.05$ was accepted as statistically significant. Analyses were performed with SPSS Software, Version 18.0 for Windows (SPSS Inc., Chicago, IL, USA).

\section{Results}

\subsection{Variation Trend of Vitamin D Status by Bonth of Age}

As shown in Figure 1A, the older infants had a higher concentration of vitamin D than younger infants $(r=0.62$, $P<0.0001)$. In addition, the overall prevalence of vitamin $\mathrm{D}$ deficiency and insufficiency were $33.80 \%$ and $18.00 \%$, respectively. There was a declining trend between vitamin $\mathrm{D}$ deficiency prevalence and month age $(r=-0.91, P<0.0001)$, so was vitamin D insufficiency $(r=-0.83, P=0.0004)$. Moreover, the prevalence of optimal vitamin D status was increased with the month age $(r=-0.91, P<0.0001)$ (Figure 1B). 

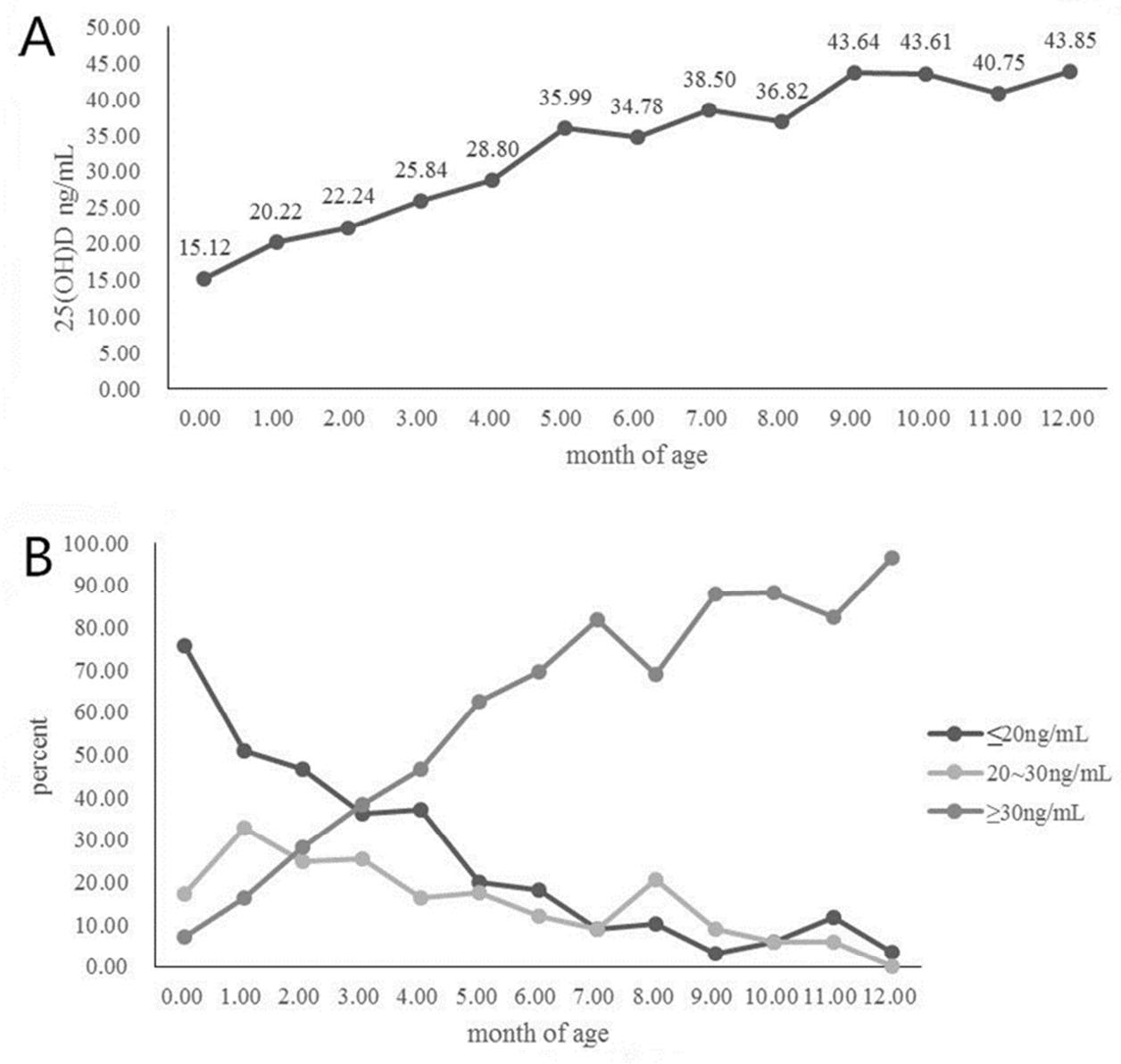

Figure 1. Vitamin D status by month of age.

\subsection{Influence Factors of Vitamin D Deficiency and Insufficiency}

Table 1 summarized the descriptive characteristics of the study population by vitamin D deficiency, insufficiency and optimal vitamin D status. There was no significant difference in sex distribution among groups $(P>0.05)$. Compared with the optimal vitamin D status group, the proportions of infant who resided in rural was significantly higher in vitamin D deficiency group $(P<0.05)$. In addition, there was a higher rate of the infant who were low birthweight, premature, breastfed in vitamin D deficiency group than optimal vitamin D status group $(P<0.05)$. Vitamin $\mathrm{D}$ deficiency group also had a higher parity and gravidity $(P<0.05)$. The distribution of vitamin D insufficiency infant was in accordance with the vitamin D deficiency group.

Table 1. Descriptive characteristics of study participants by Vitamin D status. N (\%).

\begin{tabular}{|c|c|c|c|c|}
\hline & Vitamin D deficiency $(n=169)$ & Vitamin D insufficiency $(\mathrm{n}=90)$ & optimal vitamin $D(n=241)$ & $\mathbf{P}$ \\
\hline sex & & & & 0.75 \\
\hline Boys & $105(62.13)$ & $60(66.67)$ & $156(64.73)$ & \\
\hline Girls & $64(37.87)$ & $30(33.33)$ & $85(35.27)$ & \\
\hline Region of residence & & & & $<0.0001$ \\
\hline Rural & $82(48.52)$ & $49(54.44)$ & $77(31.95)$ & \\
\hline urban & $87(51.48)$ & $41(45.56)$ & $164(68.05)$ & \\
\hline Birthweight & & & & $<0.0001$ \\
\hline$<2500 \mathrm{~g}$ & $75(44.38)$ & $16(17.78)$ & $24(9.96)$ & \\
\hline$\geq 2500 \mathrm{~g}$ & $94(55.62)$ & $74(82.22)$ & $217(90.04)$ & \\
\hline Gestational age & & & & $<0.0001$ \\
\hline premature & $82(48.52)$ & $25(27.78)$ & $30(12.45)$ & \\
\hline Term infant & $87(51.48)$ & $63(72.22)$ & $210(87.55)$ & \\
\hline Gradivity & & & & $<0.0001$ \\
\hline 1 & $85(50.30)$ & $47(52.22)$ & $151(62.66)$ & \\
\hline 2 & $37(21.89)$ & $27(30.00)$ & $70(29.05)$ & \\
\hline$\geq 3$ & $47(27.81)$ & $14(15.56)$ & $18(7.47)$ & \\
\hline Parity & & & & 0.03 \\
\hline
\end{tabular}




\begin{tabular}{|c|c|c|c|c|}
\hline & Vitamin D deficiency $(n=169)$ & Vitamin D insufficiency (n=90) & optimal vitamin $D(n=241)$ & $\mathbf{P}$ \\
\hline 1 & $91(53.85)$ & $52(59.09)$ & $160(66.67)$ & \\
\hline$\geq 2$ & $78(46.15)$ & $36(40.91)$ & $80(33.33)$ & \\
\hline Feeding patterns & & & & $<0.0001$ \\
\hline breastfeeding & $59(34.91)$ & $35(38.89)$ & $63(26.14)$ & \\
\hline Mixed feeding & $46(27.22)$ & $28(31.11)$ & $115(47.72)$ & \\
\hline Artificial feeding & $17(10.06)$ & $17(18.89)$ & $60(24.90)$ & \\
\hline Not start feeding & $34(20.12)$ & $5(5.56)$ & $2(0.83)$ & \\
\hline \multicolumn{5}{|l|}{ Maternal health in pregnant } \\
\hline Pregnancy induced hypertension & $19(11.24)$ & $4(4.44)$ & $4(1.66)$ & $<0.001$ \\
\hline Gestational diabetes mellitus & $8(4.73)$ & $5(5.56)$ & $10(4.15)$ & 0.84 \\
\hline
\end{tabular}

In our study, 41 infants were enrolled immediately after birth before feeding. Among them 34 (82.93\%) were diagnosed with vitamin D deficiency, and only 2 (4.88\%) were with optimal vitamin D status.

The selected influencing factors of vitamin D deficiency and insufficiency in infant were presented in Table 2. Based on this data, with the increase of month age, the risk of vitamin $\mathrm{D}$ deficiency $(\mathrm{OR}=0.63,95 \% \mathrm{CI}$ : 0.57-0.70) and insufficiency $(\mathrm{OR}=0.69$, 95\% $\mathrm{CI}$ : $0.62-0.77)$ were both declined. In addition, the infants resided in rural were found to be 2.29 (95\%CI: $1.34-3.93)$ and 2.55 (95\%CI: $1.45-4.50)$ times more likely to be vitamin D deficiency and insufficiency respectively, compared with the infants resided in urban. Moreover, the low birthweight infants had a high odds for vitamin D deficiency ( $\mathrm{OR}=4.04,95 \% \mathrm{CI}$ : 1.93-8.47). The artificial fed infant had the lowest odds for vitamin D deficiency $(\mathrm{OR}=0.10,95 \% \mathrm{CI}$ : 0.04-0.22) and insufficiency $(\mathrm{OR}=0.24$, 95\%CI: 0.11-0.54), compare with breastfed infants.

Table 2. Multivariate logistic regression analyses examining the influencing factors of vitamin D deficiency and insufficiency in infant.

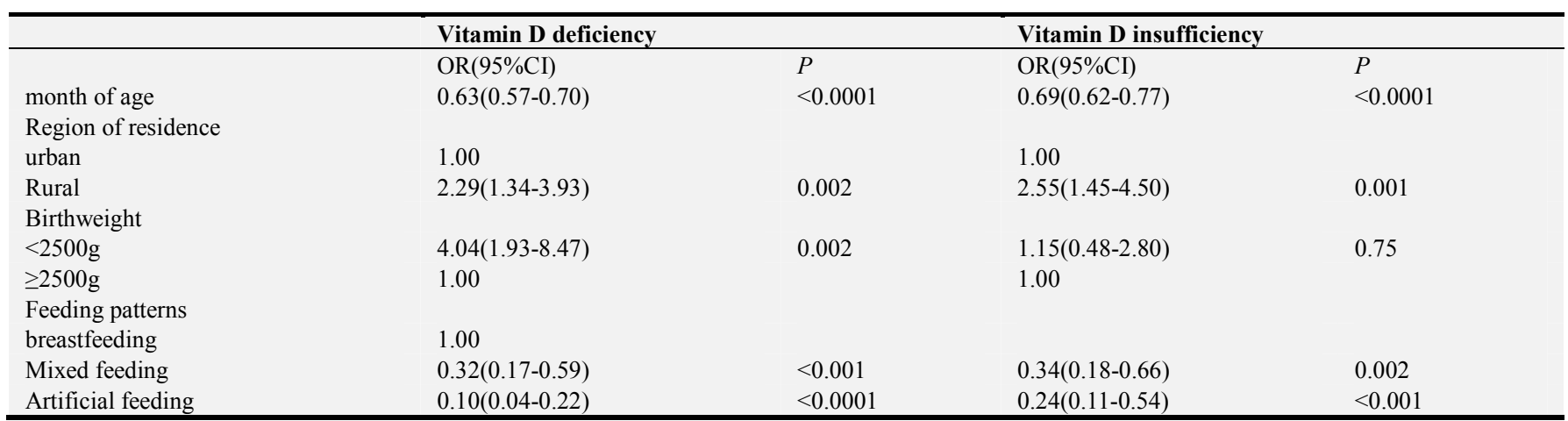

\section{Discussion}

To our knowledge, this was the first study reported the variation trend of vitamin $\mathrm{D}$ status with month age among infants. And our results showed an increased concentration of vitamin D and decreased prevalence of vitamin D deficiency and insufficiency with month age, among infants in China, during winter. This might account for the cumulative effect of routine vitamin D supplementation, increased breast milk or formula intake, the induction of complementary food and the increased ability of vitamin D synthesize and so on. Hence, along with the daily 400IU supplementation of vitamin $\mathrm{D}$, routine monitoring of vitamin D levels was very necessary, especially for infants under 4 months of age. According to the monitoring results, the vitamin D supplementary dosage would be conducted personalized and effectively.

The overall prevalence of vitamin D deficiency was 33\% in our infant group during winter, which was relatively lower compared with other age groups. In Greek primary schoolchildren, the prevalence of vitamin D lower than $20 \mathrm{ng} / \mathrm{mL}$ was $52.5 \%$ [11], and it was $45.9 \%$ in Italian children and adolescents (range 2.0-21.0 years) wintertime
[12]. The results from the occupied Palestinian territory also showed that vitamin D deficiency was more common in children older than 1 years than in children aged 1 year or younger [13]. However, $82.39 \%$ newborns at birth were vitamin D deficiency in our study, similar with the $75 \%$ in Iranian population and $84.0 \%$ in New Zealand population [14]. Another study conducted in an environment with abundant sunshine (Doha, Qatar during summer months) also found that $83 \%$ breastfed infant without vitamin D supplementation at 1 month of age were deficient [15]. Many studies had confirmed the positive relationship between maternal late pregnancy vitamin $\mathrm{D}$ concentration and newborn vitamin $\mathrm{D}$ store or cord vitamin D concentration [14, 16-18]. In addition, the results of our study also concluded that the breastfed infants owned a higher risk of vitamin D deficiency and insufficiency, consistent with the published results in other populations [15]. A double-blind randomized placebo controlled trial showed that maternal orally supplemented with $60000 \mathrm{IU}$ of vitamin D daily in the early postpartum period could increase the concentration of $25(\mathrm{OH}) \mathrm{D}$ in exclusively breasted infants at 6 months of age [19]. So for breast-fed infants, vitamin D supplementation combined with maternal postpartum supplement might have 
a better effect on vitamin D deficiency prevention. Above all, maternal prenatal to postpartum vitamin D supplementation might be an effective intervention to promote optimal vitamin $\mathrm{D}$ status in newborns and infants [20]. The serum $25(\mathrm{OH}) \mathrm{D}$ concentration in lactating mothers should at least up to the recommended target value of $>50 \mathrm{nmol} / \mathrm{L}$.

Our results also concluded that the low birth weight infant was the risk group for vitamin D deficiency. The vitamin D deficiency of low birth weight might be the subsequent effect of maternal vitamin D deficiency. Many studies concluded that maternal vitamin D insufficiency is independently associated with low birth weight [21-23]. Hence, for low birth weight infant, might be a higher dose of vitamin D supplement was effectible for vitamin D deficiency prevention, and further studies were needed to explore.

Published literature presented that vitamin D deficiency was more likely in girls than in boys, because of the lower dietary intake of vitamin D and lower levels of physical activity executed outdoors [11, 13, 24, 25]. But for infant, the dose of vitamin D supplementation was the same between different sex. In addition, during winter, the body surface area of exposure to sunlight was rare and most of the infant had no capacity for autonomous activity. Hence, there were no sex difference observed in the present study.

In spring, summer and autumn season, the urbanization degree differences on vitamin D status was concerned $[11,26]$. These differences mainly be attributed to the particularities of the physical environment which could be more supportive of increased outdoor physical activity levels and consequently greater sun exposure in rural area [27]. But in winter months (January or March), as the subjects both urban and rural regions usually spent more time indoors, due to the colder weather conditions and shorter daytime, sun exposure during this period was not efficient enough in stimulating endogenous vitamin D synthesis [28]. Manios et al did not find the regional differences on vitamin D status during winter [11]. But in the present study, the infant living in rural region were more at risk for vitamin D deficiency than those live in urban areas, consistently with the results from Chinese children and adolescents [29]. The quality of formula and vitamin D supplements might explain these difference.

The findings of the current study should be interpreted in light of its strengths and limitations. Regarding strengths, the current study presented the variation trend of vitamin D status with month age, focused on the infant in wintertime. For limitations, first was the absence of the measurement of dietary vitamin D intake. Secondly, our study was a hospital-based studies which might skew the results. The longitudinal investigations with a larger sample size, involving all seasons should be conducted in further researches.

\section{Conclusion}

In summary, in wintertime, the concentration of vitamin D for infant was increased with month age. And low birthweight, breastfed, living in rural area might be predictors of vitamin D deficiency and insufficiency. Hence, except for infant vitamin D supplementation strategies, maternal prenatal to postpartum vitamin D supplementation might be an effective intervention to promote optimal vitamin D status in infants.

\section{Author Contributions}

Wei Li, Qiong Dai and Yongying Qiu written the initial draft and revised it. Jianqiong Liu collected the data and analyzed. Yan Guo conducted the vitamin D detection, Bin Wang was the project leadership and designed the work.

\section{Conflicts of Interest Statement}

The authors report no conflict of interest.

\section{Acknowledgements}

This work was supported by Hubei Provincial Health and Family Planning Commission under Grant WJ2018H0134, WJ2018H0145, WJ2018H0167.

\section{References}

[1] Braegger C, Campoy C, Colomb V, Decsi T, Domellof M, Fewtrell $M$, et al. Vitamin $D$ in the Healthy Paediatric Population: A Position Paper by the ESPGHAN Committee on Nutrition. Journal of pediatric gastroenterology and nutrition. 2013; 56: 692-701.

[2] Taylor CL, Thomas PR, Aloia JF, Millard PS, Rosen CJ. Questions About Vitamin D for Primary Care Practice: Input From an NIH Conference. The American Journal of Medicine. 2015, 128:S0002934315005094.

[3] Cashman KD, Kiely M. Towards prevention of vitamin D deficiency and beyond: knowledge gaps and research needs in vitamin D nutrition and public health. British Journal of Nutrition. 2011, 106: 1617.

[4] Theodoratou E, Tzoulaki I, Zgaga L, Ioannidis JP. Vitamin D and multiple health outcomes: umbrella review of systematic reviews and meta-analyses of observational studies and randomised trials. BMJ. 2014; 348: g2035.

[5] Hagenau T, Vest R, Gissel T N, Poulsen CS, Erlandsen M, Mosekilde L, et al. Global vitamin D levels in relation to age, gender, skin pigmentation and latitude: an ecologic meta-regression analysis. Osteoporosis International 2009, 20: 133.

[6] Misra M, Pacaud D, Petryk A, Collett-Solberg PF, Kappy M. Vitamin D deficiency in children and its management: Review of current knowledge and recommendations. Pediatrics. 2008; 122: 398-417.

[7] Wagner CL, Greer FR. Prevention of Rickets and Vitamin D Deficiency in Infants, Children, and Adolescents. Pediatrics. 2008; 122: 1142-1152.

[8] Jie Liu, WQ Li. A survey of Vitamin D level and the rate of Vitamin D supplementation in infants and young children in Tianjin. Journal of Applied Clinical Pediatrics. 2017; 32: 1471-1474. 
[9] Rajakumar K. Vitamin D, cod-liver oil, sunlight, and rickets: a historical perspective. Pediatrics. 2003; 112: e132-e135.

[10] Fiscaletti M, Stewart P, Munns CF. The importance of vitamin $\mathrm{D}$ in maternal and child health: a global perspective. Public Health Reviews. 2017; 38.

[11] Manios Y, Moschonis G, Hulshof T, Bourhis A, Hull GLJ, Dowling KG, et al. Prevalence of vitamin D deficiency and insufficiency among schoolchildren in Greece: the role of sex, degree of urbanisation and seasonality. British Journal of Nutrition. 2017; 118: 550-558.

[12] Vierucci F, Del PM, Fanos M, Gori M, Carlone G, Erba P, et al. Vitamin D status and predictors of hypovitaminosis D in Italian children and adolescents: a cross-sectional study. Eur $J$ Pediatr. 2013; 172: 1607-1617.

[13] Chaudhry A, Hajat S, Rizkallah N, Abu-Rub A. Risk factors for vitamin $A$ and vitamin $D$ deficiencies in children younger than 5 years in the occupied Palestinian territory: a cross-sectional study. Lancet. 2018; 391 Suppl 2: S3.

[14] Kazemi A, Sharifi F, Jafari N, Mousavinasab N. High prevalence of vitamin D deficiency among pregnant women and their newborns in an Iranian population. Journal of women's health. 2009; 18: 835-839.

[15] Dawodu A, Salameh K, Al-Janahi NSA, Reedy A. Prevalence and risk factors for low vitamin D status among breastfeeding mother-infant dyads in an environment with abundant sunshine. Int J Womens Health. 2016; 8: 529-535.

[16] Dawodu A, Tsang RC. Maternal vitamin D status: effect on milk vitamin D content and vitamin D status of breastfeeding infants. Advances in Nutrition. 2012; 3: 353-361.

[17] Marshall I, Mehta R, Petrova A. Vitamin D in the maternal-fetal-neonatal interface: clinical implications and requirements for supplementation. Journal of maternal-fetal \& neonatal medicine. 2013; 26: 633-638.

[18] Emmerson A, Dockery KE, Mughal MZ, Roberts SA, Tower CL, Berry JL. Vitamin D status of White pregnant women and infants at birth and 4 months in North West England: A cohort study. Matern Child Nutr. 2018; 14.

[19] Naik P, Faridi MMA, Batra P, Madhu SV. Oral supplementation of parturient mothers with vitamin D and its effect on 25OHD status of exclusively breastfed infants at 6 months of age: A double-blind randomized placebo controlled trial. Breastfeeding medicine. 2017; 12: 621-628.
[20] Thiele DK, Ralph J, El-Masri M, Anderson CM. Vitamin D3 supplementation during pregnancy and lactation improves vitamin D status of the mother-infant dyad. J Obstet Gynecol Neonatal Nurs. 2017; 46: 135-147.

[21] Wang H, Xiao Y, Zhang L, Gao Q. Maternal early pregnancy vitamin $\mathrm{D}$ status in relation to low birth weight and small-for-gestational-age offspring. J Steroid Biochem Mol Biol. 2018; 175: 146-150.

[22] Benjamin NS, White AJ, Vidal AC, Schildkraut JM, Murtha AP, Murphy SK, et al. Maternal vitamin D, DNA methylation at imprint regulatory regions and offspring weight at birth, 1 year and 3 years. Int J Obes (Lond). 2018; 42: 587-593.

[23] Eggemoen AR, Jenum AK, Mdala I, Knutsen KV, Lagerlov P, Sletner L. Vitamin D levels during pregnancy and associations with birth weight and body composition of the newborn: a longitudinal multiethnic population-based study. $\mathrm{Br} J$ Nutr. 2017; 117: 985-993.

[24] Dong Y, Pollock N, Stallmann-Jorgensen IS, Gutin B, Lan L, Chen TC, et al. Low 25-hydroxyvitamin D levels in adolescents: race, season, adiposity, physical activity, and fitness. Pediatrics. 2010; 25: 1104-1111.

[25] Black LJ, Burrows SA, Jacoby P, Oddy WH, Beilin LJ, Chan SPW, et al. Vitamin D status and predictors of serum 25-hydroxyvitamin D concentrations in Western Australian adolescents. Br J Nutr. 2014; 112: 1154-1162.

[26] Bromage S, Rich-Edwards JW, Tselmen D, Baylin A, Houghton LA, Baasanjav N, et al. Seasonal epidemiology of serum 25-hydroxyvitamin D concentrations among healthy adults living in rural and urban areas in Mongolia. Nutrients. 2016; 8 .

[27] Lucas RM, Ponsonby AL, Dear K, Valery PC, Taylor B, van der Mei I, et al. Vitamin D status: multifactorial contribution of environment, genes and other factors in healthy Australian adults across a latitude gradient. J Steroid Biochem Mol Biol. 2013; 136: 300-308.

[28] Holick MF. Vitamin D: a d-lightful solution for health. Journal of investigative medicine. 2011; 59: 872-880.

[29] Hu Y, Chen J, Wang R, Li M, Yun C, Li W, et al. Vitamin D nutritional status and its related factors for Chinese children and adolescents in 2010-2012. Nutrients. 2017; 9. 